\title{
Sentido de vida en adultos mayores institucionalizados desde el enfoque Humanista-Existencial
}

\author{
Meaning of Life in Institutionalized Older Adults from \\ the Humanist-Existential Approach
}

\author{
César Molina Codecido ${ }^{1}$ \\ https://orcid.org/0000-0002-9480-2631 \\ ¡Unidad de Psicología de la Salud, Hospital San José, Chile
}

\begin{abstract}
Resumen. Objetivo. Identificar el sentido de vida en un grupo de adultos mayores institucionalizados, desde una perspectiva humanista-existencial. Método. Se efectuó un diseño cualitativo fenomenológico en el que se entrevistaron a 8 adultos mayores con edades entre 74 y 99 años, bajo la técnica de relato de vida, efectuándose un análisis narrativo temático. Resultados. Se observaron 3 ejes temáticos (actividades previas a la institución, historia de vida personal y acontecimientos vitales en torno al sufrimiento). De estos, se desprendieron los valores existenciales que permitieron dar cuenta de un sentido de vida en los adultos mayores institucionalizados. En conclusión, los adultos mayores orientan su vida hacia sí mismos, hacia las demás personas dentro de la institución y presentan un sentido hacia la muerte.
\end{abstract}

Palabras clave. Adulto mayor, institucionalización, sentido de vida, Psicología Humanista-Existencial

Abstract. Objective. Identify the meaning of life in a group of institutionalized older adults, from a humanistic-existential perspective. Method. A phenomenological qualitative design was carried out where eight older adults aged between 74 and 99 years were interviewed, using the life story technique, carrying out a thematic narrative analysis. Results. Three thematic axes were observed (activities prior to the institution, history of personal life, and vital events around suffering). From these, the existential values that allowed to give an account of a meaning of life in institutionalized older adults emerged. In conclusion, older adults orient their life towards themselves, towards other people within the institution, and have a meaning of death.

Keywords. Elderly, Institutionalization, Meaning of life, Humanistic-Existential Psychology

${ }^{1}$ César Molina Codecido. Unidad de Psicología de la Salud, Hospital San José, Chile. Dirección postal: Calle San José 1196. Comuna de Independencia.8380419, Santiago de Chile, Chile. E-mail: cerro.molina@gmail.com

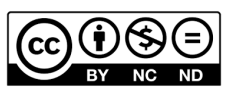




\section{Introducción}

A nivel mundial existe un aumento en la tasa de adultos mayores. Se estima que entre los años 2000 y 2050 la población mayor a 60 años aumentará desde un 11\% a un 22\%, pasando de 605 millones a 2000 millones (Organización Mundial de la Salud [OMS], 2017). Tanto en América Latina como a nivel local se observa que la población envejece cada vez más (Comisión Económica para América Latina y el Caribe [CEPAL], 2015), hecho que se debe a la disminución de la fecundidad y al aumento en la esperanza de vida (OMS, 2017).

Para afrontar los desafíos que plantea el envejecimiento a nivel internacional, se han establecido una serie de asambleas, las cuales han tenido por objetivo la salud y el bienestar de los adultos mayores (Servicio Nacional del Adulto Mayor [SENAMA], 2013). Estos objetivos incluyen factores fisiológicos, psicológicos, sociales y espirituales, que influyen en el envejecimiento de las personas (Al-Nasa'h, 2020; Caldas et al., 2020; Cassady et al., 2020; D'cruz \& Banerjee, 2020; Howell \& Peterson, 2020; Lang et al., 2020; da Silva et al., 2020; Reich et al., 2020; Sexton et al., 2020; Timbers \& Childers, 2020; Worrall et al., 2020).

La evidencia a nivel internacional señala que una de las variables que aumentan el bienestar en la adultez mayor es el sentido de vida y el propósito de vida (Ju, 2017; Ju et al., 2013; Steger et al., 2009; Volkert, 2017), lo cual incluso puede generar una reducción en el riesgo de mortalidad (Hill \& Turiano, 2014; Kim et al., 2013). De acuerdo con Krause (2009) los adultos mayores con propósitos de vida perciben su salud de manera más positiva y experimentan menos limitaciones funcionales, mientras que el sentido de vida se asocia a un mayor bienestar y a la facilitación de los procesos de afrontamiento ante situaciones estresantes (Park \& Folkman, 1997). Por otro lado, Frankl (1995) plantea que los adultos mayores con un sentido de vida establecido se mantienen a nivel psíquico e intelectual y les permite preservarse de las enfermedades.

La búsqueda de sentido en el ser humano aparece a lo largo de todas las etapas de la vida debido a su condición de ser inacabado y a que la misma existencia debe ser plasmada o sobrellevada en cada momento (Längle, 2008). De acuerdo con Frankl (1979) lo que importa no es el sentido de vida de manera general, sino el significado concreto de cada persona en un determinado momento. De acuerdo con Längle (2008) el sentido se entiende como "una manera especial de plasmar la situación" (p. 11). Esto quiere decir que el ser humano constantemente se encuentra descubriendo su sentido de vida, dado que siempre estará cambiando con el paso del tiempo (Frankl, 1979).

A partir de la vida misma, el sentido se puede descubrir a través de tres modos: al realizar una acción, al tener algún principio que la oriente y por el sufrimiento ante las crisis que puedan aparecer (Frankl, 1979). Estos modos son planteados a partir de tres categorías de valores denominados como las vías principales hacia el sentido (Frankl, 1983), ya que el sentido se da al momento de realizar uno de estos valores (Vial, 2000), los cuales se clasifican en:

- Valores creativos: estos valores aparecen cuando el ser humano logra generar algo en el mundo, logra dar al mundo o produce en él. En este caso, se entregan elementos valiosos en el mundo, enriqueciéndolo con esta acción (Längle, 2008).

- Valores vivenciales: estos valores permiten acoger los elementos importantes de la vida, donde el ser humano recibe algo del mundo a través de su propia experiencia (Längle, 2008).

- Valores actitudinales: el ser humano al no estar con frecuencia ante situaciones que conlleven situaciones de sufrimiento, debe tener una posición personal frente a los acontecimientos vitales ineludibles que conllevan hacia él. Ante esto, lo primero que ocurre es estar en 
una situación de difícil afrontamiento, para después aclarar lentamente el panorama ocurrido, por ejemplo, la muerte. Lo más importante es la decisión que se toma frente a este suceso, pues se adopta una posición en la que se sigue viviendo (Längle, 2008).

En este aspecto, el tema de la muerte aparece en el ser humano como un acontecimiento difícil de afrontar y se desplaza a lo largo del tiempo, esto a pesar de que cualquier momento es el momento para morir (Längle, 2008). La muerte es mayormente aceptada a medida que se avanza en edad, pero aun así tiene un lugar apartado de lo cotidiano, existiendo una muerte institucional (ya sea en geriátricos u hospitales), más que una muerte personal.

En este sentido, la mayoría de los adultos mayores con sentidos de vida pueden llegar a tener un envejecimiento saludable, sin embargo, el contexto en el cual se lleve a cabo este proceso también influirá en su calidad de vida (Wiles, 2005), por tanto, no es lo mismo envejecer en la propia casa, a envejecer en un Hogar residencial (Aydın et al., 2020; Tiwari et al., 2016; Worrall et al., 2020).

En los Hogares Residenciales, los adultos mayores pueden presentar un aceleramiento en su deterioro físico (Freitas \& Scheicher, 2010), un mayor nivel de dependencia con respecto a sus actividades de la vida diaria (Baltes \& Wahl, 1990; Moreira et al., 2016) y mayores factores de riesgo para la salud mental, tales como el aislamiento, la soledad o el déficit en su cuidado personal (Brownie \& Horstmanshof, 2011; Theurer, et al., 2015). Asimismo, esta situación contrasta con investigaciones que han comparado los índices de depresión entre adultos mayores institucionalizados y no institucionalizados, en el que los primeros presentan mayores niveles de depresión (Azeem \& Naz, 2015; Silva et al., 2015).

Al considerar las ventajas que trae consigo el descubrir un sentido de vida, se han observado estudios que describen el sentido de vida en adultos mayores institucionalizados, destacándose que, el hecho de poder vivir el día a día, el recibir cuidados adecuados y mantener buenas relaciones interpersonales genera un sentido de vida en dichas personas (Elsherbiny \& Al Maamari, 2018; Flecha, 2019; Franco, 2018; Usta \& Bayram, 2021).

Sin embargo, si bien los estudios refieren de forma implícita la presencia de los valores existenciales en el sentido de vida, no se ha observado una profundización explícita en torno a los mismos. Por este motivo, el identificar el sentido de vida mediante los valores existenciales, permitirá ahondar la forma en cómo logran el bienestar los adultos mayores institucionalizados.

Ante esto, emerge como pregunta de investigación ¿cuál es el sentido de vida que poseen los adultos mayores institucionalizados? Así mismo, el objetivo es identificar el sentido de vida en un grupo de adultos mayores institucionalizados, desde una perspectiva humanista-existencial.

\section{Método}

Tipo de estudio

Se optó por una metodología cualitativa puesto que permite acceder a los procesos subjetivos y a la perspectiva que tiene el grupo al cual se investigará (Flick, 2004). Se escogió un diseño fenomenológico, con el fin de realizar un análisis de las experiencias significativas de las personas entrevistadas que permite conocer la historia de vida de ellas, unidas a sus vivencias y relatos personales (Guillen \& Elida, 2019). 


\section{Participantes}

El campo de estudio estuvo compuesto por adultos mayores usuarios de dos Establecimientos de Larga Estadía (ELEAM) en la ciudad de Santiago, Chile, donde se albergaban a 100 adultos mayores en uno y a 95 adultos mayores en otro. Dichos establecimientos son de carácter mixto y dependientes de una Fundación Benéfica, la cual acoge a adultos mayores sobre los 60 años, con un grado de dependencia parcial o severa, sin poseer los cuidados adecuados para permanecer en su medio habitual. Las personas fueron seleccionadas mediante un muestreo por conveniencia, en el que se llegó a un total de 8 personas, 1 hombre y 7 mujeres, con una edad comprendida entre los 74 años y los 99 años (Tabla 1). Dicho número de participantes permitió completar la comprensión de las temáticas a estudiar a través de la saturación teórica de las narrativas obtenidas (Strauss \& Corbin, 2002). Las personas entrevistadas debían tener desde 60 años en adelante, no presentar algún tipo de demencia y tener más de 6 meses de estadía al interior de la institución, esto con el fin de que ya tuviesen un proceso de adaptación en cuanto a los horarios y lugares de la residencia en la cual se encontraban (Wiles, 2005).

Tabla 1

Identificación y características de las personas entrevistadas

\begin{tabular}{ccccc}
\hline Código & Participante & Sexo & Edad & Tiempo en la Institución \\
\hline E1 & Entrevistada 1 & Mujer & 99 & 2 años \\
E2 & Entrevistada 2 & Mujer & 84 & 2 años \\
E3 & Entrevistado 3 & Hombre & 83 & 5 años \\
E4 & Entrevistada 4 & Mujer & 81 & 1 año con tres meses \\
E5 & Entrevistada 5 & Mujer & 84 & 5 años \\
E6 & Entrevistada 6 & Mujer & 74 & 3 años \\
E7 & Entrevistada 7 & Mujer & 77 & 5 años \\
E8 & Entrevistada 8 & Mujer & 70 & 8 meses \\
\hline
\end{tabular}

\section{Instrumentos}

Se elaboraron entrevistas en profundidad, bajo el formato de relatos de vida, para abordar las temáticas relacionadas con las historias de vida de cada adulto mayor y su vínculo con un sentido de vida. El formato de las preguntas para dichas entrevistas fue elaborado a partir de las recomendaciones de Riessman (2008), quien plantea tres elementos a considerar: la práctica narratológica (el impulso narrativo), el relato a narrar (constituyéndose el dato narrativo) y el análisis narrativo (centrándose en cómo es construida la narrativa y el contexto donde se sitúan tales experiencias). Para la validación del contenido del instrumento, se sometió al juicio de dos jueces expertas.

La premisa del instrumento consistió en la pregunta guía inicial ¿cuáles han sido las experiencias más importantes en su vida?, para luego profundizar bajo las temáticas de historia de vida personal, actividades previas a la institución y acontecimientos vitales en torno al sufrimiento (Tabla 2). 
Tabla 2

Preguntas de entrevista narrativa según área temática

\begin{tabular}{|c|c|c|}
\hline Tipo de pregunta & Eje temático & Pregunta tipo \\
\hline \multirow[t]{2}{*}{ Pregunta guía } & $\begin{array}{l}\text { Historia de } \\
\text { vida personal }\end{array}$ & $\begin{array}{l}\text { 1. ¿Cuáles han sido las experiencias } \\
\text { más importantes en su vida? }\end{array}$ \\
\hline & & 2. ¿Qué cosas hace usted aquí en el hogar? \\
\hline \multirow[t]{3}{*}{$\begin{array}{l}\text { Preguntas de } \\
\text { profundización }\end{array}$} & $\begin{array}{l}\text { Actividades previas } \\
\text { a la Institución }\end{array}$ & $\begin{array}{l}\text { 1. ¿Qué actividades importantes } \\
\text { ha tenido en su vida? }\end{array}$ \\
\hline & $\begin{array}{l}\text { Acontecimientos } \\
\text { vitales en torno }\end{array}$ & $\begin{array}{l}\text { 1. ¿Cómo ha podido sobreponerse } \\
\text { a los momentos dificiles? }\end{array}$ \\
\hline & al sufrimiento & 2. ¿Qué piensa usted sobre la muerte? \\
\hline
\end{tabular}

\section{Procedimiento}

En un primer momento, se indagó en un marco teórico que permitiera enmarcar la construcción y validación de la pregunta inicial y de las temáticas a tratar durante la recolección de la información. Para ello, se analizaron los postulados del enfoque humanista-existencial, junto con las bases metodológicas de la técnica de relato de vida. Al terminar la construcción del instrumento, se solicitó a dos expertas (una investigadora en el área humanista-existencial y otra especialista en métodos narrativos de investigación) las validaciones en cuanto al contenido y la estructura del instrumento, las cuales fueron, por un lado, con base en los postulados de Frankl (1979) y Längle (2008) en cuanto al humanismo-existencial, y por otro, a Riessman (2008).

Paralelamente, se contactó a la encargada zonal de la institución, con quien se establecieron acuerdos específicamente orientados hacia los aspectos éticos de la investigación. Una vez efectuado el contacto, se procedió a contactar a los adultos mayores que estuvieran interesados en la investigación de manera voluntaria, realizándose un muestreo por conveniencia.

Para el proceso de entrevistas, se desarrolló una primera entrevista piloto, con grabadora de audio, la cual se llevó a cabo en dos sesiones y en una habitación del Hogar residencial habilitada para ello. La entrevista piloto se elaboró con el fin de analizar si la pregunta guía inicial era lo suficientemente clara y si la forma de profundizar en los ejes temáticos era adecuada. Después de la primera sesión de la entrevista piloto, esta fue transcrita y analizada, junto con una experta en metodología cualitativa narrativa, en torno a los ejes temáticos y la forma en cómo se abordaron las preguntas, generando además una triangulación de la narrativa obtenida. Esto permitió sistematizar la forma de análisis de la primera sesión, para luego, en la segunda sesión, validar el contenido de las temáticas tratadas con la persona entrevistada en la primera sesión. Después de tal validación, se continuó con la segunda sesión. Posterior a esto, se analizó nuevamente con una experta los ejes temáticos, para así continuar con la segunda parte de la triangulación de la narrativa, junto con la sistematización de la forma para efectuar las demás entrevistas. 
El resto de las entrevistas se realizaron en una habitación acondicionada para efectuarlas. Cada entrevista fue dividida en dos sesiones por cada entrevistado, con grabadora de audio y con una duración entre 35 y 80 minutos por sesión, con un promedio de cincuenta minutos por cada una. Dichas entrevistas fueron realizadas durante un período de cuatro semanas para un total de 8 personas. En la primera sesión, se abordaron aspectos generales de la vida de cada entrevistado, mientras que en la segunda sesión (con la primera sesión transcrita) se dialogó la narrativa de la primera sesión con cada persona entrevistada, para así validar, profundizar y aclarar las áreas señaladas en la entrevista anterior.

En cuanto a los aspectos éticos de la investigación, se le entregó a cada uno de los entrevistados un Consentimiento Informado, el cual fue aprobado por el Comité de Ética de la Universidad de Chile, quien toma como referente la Declaración Universal de Bioética y Derechos Humanos de la UNESCO. En dicho documento se explicaron los objetivos del estudio, la naturaleza de las entrevistas, la confidencialidad de la información obtenida y el carácter voluntario de la participación de cada persona mayor, pudiendo abandonar en cualquier momento la investigación y respetando su autonomía en todo momento. Además, para mayor protección de los datos, los audios de las entrevistas fueron encriptados, manteniendo en el anonimato la identidad de los participantes.

\section{Estrategia de análisis}

Para el análisis de la información se realizó un análisis narrativo de las entrevistas, dado que permite observar cómo la persona construye una narración respecto a sí misma y su vida (Capella, 2013). Esta forma de análisis se centra en el contenido de la narrativa y en la forma en cómo es construida (Capella, 2013), por lo que el contexto que rodee al individuo incidirá en las experiencias que construya en torno a sí mismo y los otros. Particularmente, se realizó un análisis narrativo temático, en el cual el contenido de las entrevistas fue el foco principal (Riessman, 2008), incorporando los temas que organizan la narración, la secuencia de eventos y las personas involucradas, entre otros (Capella, 2013). En este aspecto, la narrativa, aparte de reconstruir los hechos y las vivencias de las personas entrevistadas, genera una producción que crea un sentido acerca de la propia historia (Bernasconi, 2011).

En este tipo de análisis, la relación entre investigador-investigado se torna sumamente importante, ya que lo que ocurre en ese vínculo intersubjetivo genera un conocimiento adicional, esto debido a que el entrevistado narra hacia alguien que escucha y observa, emergiendo un relato con una voz singular y hacia un oído en particular, es decir, con una intencionalidad (Cardona \& Salgado, 2015).

De este modo, se observaron los contenidos que emergieron a partir de cada entrevista, para analizar los temas y dar cuenta de las principales temáticas surgidas en cada persona entrevistada (Capella, 2011), organizando las entrevistas posteriormente con base en ejes temáticos.

\section{Resultados}

A continuación, se presentan los resultados de las 8 personas entrevistadas (desde ahora con un código de identificación "E" junto con un número) con base en los ejes temáticos abordados (Tabla 3). 
Tabla 3

Ejes temáticos narrativos de las personas entrevistadas

\begin{tabular}{|c|c|c|c|}
\hline Eje temático & Definición & $\begin{array}{l}\text { Subtema del } \\
\text { eje temático }\end{array}$ & Ejemplo \\
\hline $\begin{array}{l}\text { Actividades } \\
\text { previas a la } \\
\text { Institución }\end{array}$ & $\begin{array}{l}\text { Refiere a las acciones } \\
\text { que realizaban a diario } \\
\text { las personas cuando } \\
\text { estaban fuera de la } \\
\text { Institución, con el fin de } \\
\text { enriquecer o entregar } \\
\text { algo beneficioso } \\
\text { hacia otra persona. }\end{array}$ & $\begin{array}{l}\text { Hechos } \\
\text { familiares }\end{array}$ & $\begin{array}{l}\text { "...un telar para hacer bufandas, } \\
\text { si, entonces ahí empecé a } \\
\text { trabajar...y vendí y empecé a } \\
\text { hacer y me pedían..." (E4) } \\
\text { "...cuando nos casamos, me dijo } \\
\text { "yo viajo bastante, porque voy } \\
\text { a hacer conferencias", entonces } \\
\text { me dijo "como la voy a dejar } \\
\text { sola, le llevo, vamos"... "ya" le } \\
\text { dije yo..." (E2) }\end{array}$ \\
\hline \multirow[t]{2}{*}{$\begin{array}{l}\text { Historia de } \\
\text { vida personal }\end{array}$} & \multirow{2}{*}{$\begin{array}{l}\text { Refiere a las historias } \\
\text { de vida que señalaron } \\
\text { las personas } \\
\text { institucionalizadas }\end{array}$} & $\begin{array}{l}\text { Vida anterior a } \\
\text { la Institución }\end{array}$ & $\begin{array}{l}\text { "...mis nietos, mis hijas...ellos } \\
\text { tienen su profesión, y eso me da } \\
\text { una satisfacción..." (E3) }\end{array}$ \\
\hline & & $\begin{array}{l}\text { Vida al interior } \\
\text { de la Institución }\end{array}$ & $\begin{array}{l}\text { "...yo todos los días, me levanto } \\
\text { a las } 5 \text { de la mañana voy al } \\
\text { comedor de la esquinita...y se } \\
\text { ve el amanecer...yo disfruto el } \\
\text { amanecer porque ninguno es } \\
\text { igual, todos son distintos..." (E2). }\end{array}$ \\
\hline \multirow[t]{2}{*}{$\begin{array}{l}\text { Acontecimientos } \\
\text { vitales en torno } \\
\text { al sufrimiento }\end{array}$} & \multirow[t]{2}{*}{$\begin{array}{l}\text { Refiere a la forma en } \\
\text { cómo las personas } \\
\text { institucionalizadas } \\
\text { afrontan los sucesos } \\
\text { pasados en el } \\
\text { momento presente, o } \\
\text { también pensando a } \\
\text { futuro, específicamente } \\
\text { en cuanto a la muerte. }\end{array}$} & $\begin{array}{l}\text { Actitud frente a } \\
\text { acontecimientos } \\
\text { vitales }\end{array}$ & $\begin{array}{l}\text { "mi doctora me dijo "usted no } \\
\text { va a poder caminar, porque ese } \\
\text { lado del cerebro está muerto" } \\
\text {... pero nunca diga usted "no } \\
\text { puedo", siempre va a poder, en } \\
\text { algo va a poder, y nunca deje de } \\
\text { decirlo" (E2) }\end{array}$ \\
\hline & & $\begin{array}{l}\text { Actitud hacia } \\
\text { la muerte }\end{array}$ & $\begin{array}{l}\text { "y yo le pido a mi Dios que } \\
\text { me deje llegar a los } 100 \text { [años], } \\
\text { y después que haga lo que } \\
\text { quiera" (E1) }\end{array}$ \\
\hline
\end{tabular}

\section{Actividades previas al ingreso en la institución}

Las actividades previas a la institucionalización de los entrevistados refieren a las actividades importantes que hicieron durante su vida, pero con un énfasis en cuanto al objetivo que buscaban. Se observa que las actividades implicaron dos subtemas: el trabajo y los hechos familiares.

En relación con el área del trabajo, se vio como un elemento que permitió mantener económicamente a la familia. Además, el trabajo fue visto como una posibilidad de entregar algo hacia las demás personas, recuerdan la gratificación al hacer dicha labor, al generar un mayor bienestar consigo mismo en la actualidad, como cuando una de las entrevistadas señaló "Yo fui una gran basquetbolista, y una maestra de cocina, de p-r-i-m-e-r-a...de todo, repostería y cocina... yo tenía 5 ayudantes...gané mucha plata" 
(E1), mientras que otras comentaban "trabajar 12 años en mecánica dental...yo les di mucho, mucho, a mucha gente, sonrisas... incluso, a la que no tenía como pagar, yo, de mi bolsillo, le pagaba los materiales" (E2), también, mencionaron aspectos vinculados con objetivos de vida "siempre quise hacer lo que pensé, porque de chica, de niña chica, me gustaba la peluquería...le cortaba el pelo a unos vecinitos míos con una máquina de mi mamá para cortar ropa" (E4). Estas narrativas dieron cuenta de lo reconfortante que fue para las personas entrevistadas el entregar un servicio hacia las demás personas.

En cuando a los hechos familiares, estos aparecieron durante las entrevistas, dando cuenta de que no necesariamente debían existir eventos extraordinarios para ser recordados, sino que más bien en su cotidianidad encontraron los acontecimientos significativos, como cuando una entrevistada señaló que "bailábamos tango con mi marido, yo era tanguera y cantábamos tango, pero no en cualquier parte, en la casa no más" (E1), mientras que otras aludían a recuerdos de la infancia, por ejemplo "sí, había carros [de trenes], en los que iba al colegio, me iban a buscar y me llevaban en carro para la casa" (E6). Además, todas las personas entrevistadas marcaban como un hito significativo "el nacimiento de los hijos, es lo más grande que he tenido, eso es impagable, cuando llega la guagua y te la entregan, ya eso es lo máximo, así que eso sería, el amor a mis hijos" (E6).

En este sentido, se observa que los valores creativos emergieron a nivel laboral al momento de entregar algo hacia las demás personas a través del trabajo, mientras que los valores vivenciales aparecieron en la vida familiar, donde el estar con una pareja o el nacimiento de un hijo fueron aspectos gratificantes ofrecidos desde la vida, hacia los entrevistados.

Historia de vida personal

Esta temática refiere a los acontecimientos significativos que señalaron los adultos mayores con respecto a su vida personal. Se desprenden dos subtemas, los cuales son la vida anterior a la institucionalización y la vida al interior de la institución.

Vida anterior a la Institución. En las entrevistas, destacan temáticas significativas relacionadas con el aspecto religioso en cuanto al reconocimiento de los demás hacia los entrevistados, tales como:

Mis rosarios están en Roma...yo trabajé con una [persona] adinerada... entonces me dijo 'la que rece más en el día, esos rosarios van a ir a Roma'... entonces recé todo el día, y tenía que escribirlo en un sobre, y... el Padre lo llevó a Roma (E1).

Mientras que otras temáticas estuvieron vinculadas con satisfacciones personales. Por ejemplo, una entrevistada comentó que una "satisfacción importante fue cuando me vi nominada en el diario, cuando había quedado aceptada en periodismo, porque yo pensaba que no" (E8). En este aspecto, aparecen acciones vinculadas con los logros personales de las personas entrevistadas, lo cual puede ser visto como un valor creativo al realizar una acción como el rezar, o también como un valor vivencial al recibir un reconocimiento de las demás personas, lo cual generó satisfacción.

Vida al interior a la Institución. Dentro de la Institución los adultos mayores también manifiestan tener experiencias de vida que les permitían vivir el día a día, ya sea mediante las visitas familiares "mi hijo viene a verme todos los fines de semana" (E6). Otra de las experiencias está dada por la capacidad para conectarse con Dios para ayudar a los demás, en este caso una entrevistada refiere "yo tenía que pedir que Dios iluminara la familia, adonde estaba, donde lo tenían, para avisar, y a los 20 días avisaron donde lo tenían" (E1), así como también la ayuda directa "yo me siento bien, porque yo como le digo, estuve 
siempre pendiente de los que necesitan ayuda, y ayudarlos, de estar ahí" (E7). En este aspecto, los sucesos cotidianos forman parte de los momentos significativos de las personas entrevistadas, ya sea a nivel familiar, religioso o social (ayudar a los demás), lo cual puede ser vinculado con los valores creativos (entregar ayuda) y vivenciales (la visita de los hijos).

\section{Acontecimientos vitales en torno al sufrimiento}

Actitud frente a acontecimientos vitales. Los adultos mayores plantean el sufrimiento en torno a una pérdida en determinado momento, ya fuese la pérdida de un hijo "tuve una tristeza bien grande porque yo perdí un hijo, y no pude tener nunca más familia" (E2), la pérdida de uno de los padres "en el caso de mi madre, su fallecimiento, es un sufrimiento que no termina nunca" (E4), la pareja "nos casamos... y después me fue pescando el alcohol... ella, recibía humillaciones...se apartó de mí" (E3) o la pérdida de autonomía:

Yo estaba agachá tomando fotos... y sentí un golpe en la cabeza [un proyectil] y caí y después de 72 días desperté en neurocirugía... eso sí que fue horrible, no poder andar, yo siempre que fui tan independiente, tan dueña de lo mío... y estar dependiendo de mis padres (E8).

Estos hechos generaron tristeza en un inicio, sin embargo, se observa en los entrevistados los valores actitudinales que permitieron afrontar dichas pérdidas resignificando su experiencia "no llores, tu hijo es un angelito, y está arriba, y te está esperando -me dijo la Madre-, está mejor que aquí" y yo ahí sentí algo muy bonito, me corría por las venas, una cosa tibiecita y alegre" (E2). En cuanto a otras pérdidas, como la separación, uno de ellos comentó:

Después mi amigo... me dijo "vamos compadre, tome lo que quiera y tome [alcohol]" ... me dice "mi comadre va a buscar uno [otra pareja], después, se va a aprovechar de ella, y se va a aprovechar de su hija, y usted será el culpable" ... me entró [lo que él dijo], me tapé el oído y me quedó (E3).

O la pérdida de facultades físicas "Dios da la mejor vida, y con eso me mantengo" (E4), "lo más que le agradezco a Dios es que no perdí la mente ni la vista, porque son órganos vitales" (E8), lo cual permite evidenciar la forma en cómo los valores actitudinales emergen ante situaciones difíciles en la vida.

Actitud hacia la muerte. Se observa una postura en la que la mayoría de las personas entrevistadas ve la muerte como una transición hacia otro estado, lo cual se ve reflejado al señalar "no creo en la muerte, muerte, yo creo que uno se va y queda en el ente, por ahí dando vueltas, en el aire... sin preocupación, uno vive como pájaro, jfantástico!" (E5). Por otro lado, también es vista como un medio para evitar enfermedades posteriores "por eso le digo a Dios que me lleve si me comienzan a dar las crisis...que le llaman... Alzheimer, que es lo más común" (E5). Se desprende de esto los valores actitudinales que permiten tomar una posición personal activa frente a la muerte.

\section{Discusión}

Se observa que el sentido de vida de los adultos mayores institucionalizados está dado por el vivir su día a día de manera tranquila y autónoma, cuando ayudan en lo posible a las personas que lo necesiten y realizan las actividades que les generan satisfacción al interior de la institución, lo cual también fue hallado en otros estudios (Elsherbiny \& Al Maamari, 2018; Flecha, 2019). Los resultados se asocian a los valores creativos y vivenciales de los entrevistados. Además, se evidencia la importancia que cada adulto mayor le 
otorga al hecho de mantenerse autovalente, para así decidir sobre su propia vida (Längle, 2008; Frankl, 1979), en la que toman un valor actitudinal frente a las situaciones que ocurren al interior de la institución.

En esta misma línea, los valores de la existencia humana fueron claves para establecer un sentido de vida en las experiencias al interior del Hogar. Los valores creativos se encontrarían relacionados con la entrega de ayuda hacia las demás personas al interior del Hogar, lo cual se vincula con el poder entregar algo hacia los demás (Frankl, 1979). En la misma línea, los valores vivenciales se experimentaron al momento de establecer un contacto con las demás personas (E4) o en el hecho de observar las maravillas de la naturaleza (E2). En este sentido, se observa que los adultos mayores refieren en algunos casos la belleza de lo que les rodea, mientras que también aluden a los valores vivenciales al hablar sobre los vínculos que poseen con otros adultos mayores (E7). Los valores vivenciales son percibidos como una oportunidad para acoger lo cotidiano (Vial, 2000) y vivir ese momento en concreto, absortos en las relaciones con los demás o en lo que entrega el entorno (Elsherbiny \& Al Maamari, 2018). Dentro de los recuerdos sobre la historia de vida de las personas entrevistadas, había algo que entregaban hacia los demás, pero que también recibían, observándose los valores creativos y vivenciales a la base (Längle, 2008). Estos valores tuvieron como fin el generar un bienestar hacia sí mismas y en las demás personas, ya fuese a nivel familiar o laboral. Además, el hecho de tener hijos es un tema que destaca como un valor vivencial en la historia de vida, lo cual incorpora un recuerdo que genera bienestar.

Los valores actitudinales emergieron cuando comenzaron a aparecer acontecimientos en la línea del sufrimiento (Elsherbiny \& Al Maamari, 2018; Frankl, 1979; Vial, 2000) cuando se tomó una decisión en un momento trágico en sus vidas. Esa decisión fue tomada a partir de la "libertad condicionada" en la que se encontraban (Frankl, 1982) la cual configuró parte de su vida ante la opción escogida. Se observa en cada relato, que la opción escogida fue la que generó mayor bienestar, dadas las condiciones contextuales. Este tema es uno de los que refleja mayor búsqueda de sentido en los entrevistados, esto dado en un inicio por la pérdida en la que emergieron los valores actitudinales posterior al evento trágico (muerte, separación o pérdida). Se observa que en la mayoría de los casos el área religiosa-espiritual tuvo un rol en la forma que tenían para sobrellevar algunas situaciones, ya fuese una enfermedad incurable o al utilizar una silla de ruedas. Como resultado, lograron adoptar una posición frente a su situación vital actual, la dotaron de un sentido que les permitía continuar con su vida en la Institución y asumieron el sufrimiento ante la enfermedad, lo cual permitía sobrellevarla. Sin embargo, es importante destacar que son situaciones difíciles de afrontar (Längle, 2008), ya que en algunos casos estuvieron durante años tratando de lograr una forma de resignificar la situación. Tal como ocurrió con la E2, quien, si bien logró continuar con su vida después de la muerte de su hijo, al interior del Hogar logró encontrar un sentido a esa muerte. Con respecto a este tema, se observa un valor actitudinal en la postura que tienen los adultos mayores con respecto a la temática de la muerte. Ellos la observan como otro estado distinto del actual e incluso como una opción para no contraer enfermedades neurodegenerativas. En este sentido, se observa que no existe un miedo a la muerte propiamente como tal, sino que se posiciona de manera positiva al relacionarse con un "pasaje" hacia otro plano de la existencia, en el que se puede estar mejor.

De esta manera, se logra evidenciar que si bien los valores de tipo creativo y vivencial permitieron hallar un sentido de vida con base en los recuerdos de la vida en general y 
al interior de la institución, los valores de tipo actitudinal son los que generan un mayor compromiso frente a la vida cotidiana de los adultos mayores, ya que frente a algún acontecimiento en el que solo se encuentre el sufrimiento, estos valores permitirán otorgar un sentido a la vida desde el dolor del ser humano (Vial, 2000). Además, se evidencia cómo estos valores que permiten el sentido de vida se vinculan con el bienestar de los adultos mayores, dado que se busca una situación que genere una satisfacción vital con respecto a la experiencia vivida (Steger et al., 2009). En este aspecto, los adultos mayores mantienen una satisfacción personal en su vida cotidiana, porque observan la vida con más alegrías que tristezas y deciden la manera en cómo vivirán su estadía al interior de la institución desde ese contexto.

Por una parte, la presente investigación complementa los hallazgos encontrados en otros estudios (Elsherbiny \& Al Maamari, 2018; Flecha, 2019; Franco, 2018; Usta \& Bayram, 2021) en cuanto a los valores existenciales a partir de las narrativas señaladas por los entrevistados. Además, se añaden los valores actitudinales hacia la muerte, el sufrimiento y las enfermedades por las cuales atraviesan las personas mayores institucionalizadas, lo cual también permite hallar un sentido de vida.

Por otro lado, al observar las fortalezas de esta investigación, se encuentra el uso de la técnica de relatos de vida desde el enfoque narrativo, la cual permitió desarrollar las entrevistas en dos sesiones, hecho que facilitó el profundizar en las temáticas mencionadas por los adultos mayores durante la primera sesión. Además, el hecho de poder narrar su propia historia fue una experiencia grata y amena para los entrevistados, de acuerdo a lo manifestado por los mismos una vez terminadas las sesiones, por lo que se podría decir que este tipo de técnicas, además de recabar información, generan bienestar en los entrevistados, lo que beneficia a los participantes durante las entrevistas.

Dentro de las limitaciones de esta investigación se puede mencionar que hubo la participación de solo un hombre en el estudio, por tanto, se recomienda para futuras investigaciones incluir un número mayor de adultos mayores hombres, con la finalidad de obtener una perspectiva dada desde el área masculina de la adultez mayor.

En cuanto a limitaciones metodológicas, se puede señalar el haber realizado los análisis por solo un investigador. Aunque se contó con expertas en la materia para asesorar las líneas metodológicas y los análisis de las entrevistas, la investigación se hubiese enriquecido aún más con otros investigadores dentro del estudio. De este modo, se recomienda que en futuras investigaciones se aumente el número de investigadores dentro de la temática, por medio de la incorporación de otros especialistas para dar una visión global frente al fenómeno de estudio. Las proyecciones en esta investigación se enmarcan en las intervenciones psicológicas desde la perspectiva humanista-existencial. Asimismo, se desarrollan métodos que les permiten a los adultos mayores encontrar un sentido a su vida cotidiana en una institución, para fomentar el bienestar de sí mismos. En este punto, el visibilizar las actividades significativas de cada adulto mayor, el ampliar la cantidad de experiencias positivas que presenten los adultos mayores al interior de la institución y el permitir acciones que fomenten la autonomía de sí mismos (a pesar de las condiciones físicas en las cuales se encuentren), facilitará el encontrar un sentido de vida que genere un mayor bienestar. 


\section{Referencias}

Al-Nasa'h, M. (2020). A spiritual-based intervention in enhancing factors of well-being among older adults in home-based care. Journal of Religion, Spirituality \& Aging. 1-22. https://doi.org/10.1080/15528030.2020.1818160

Aydın, A., Işık, A., \& Kahraman, N. (2020). Mental health symptoms, spiritual well-being and meaning in life among older adults living in nursing homes and community dwellings. Psychogeriatrics. https://doi.org/10.1111/psyg.12613

Azeem, F., \& Naz, M. (2015). Resilience, Death Anxiety, and Depression Among Institutionalized and Noninstitutionalized Elderly. Pakistan Journal Of Psychological Research, 30(1), 111-130.

Baltes, M., \& Wahl, H. (1990). Dependencia en los ancianos. Martínez-Roca.

Bernasconi, O. (2011). Aproximación narrativa al estudio de fenómenos sociales: Principales líneas de desarrollo. Acta Sociológica, 1(56), 9-36. http://dx.doi. org/10.22201/fcpys.24484938e.2011.56.28611

Brownie, S., \& Horstmanshof, L. (2011). The management of loneliness in aged care residents: An important therapeutic target for gerontological nursing. Geriatric Nursing, 35(5), 318-325. https://doi.org/10.1016/j.gerinurse.2011.05.003

Caldas, V., Fernandes, J., Vafaei, A., Gomes, C., Costa, J., Curcio, C., \& Guerra, R. O. (2020). Life-Space and Cognitive Decline in Older Adults in Different Social and Economic Contexts: Longitudinal Results from the IMIAS Study. Journal of cross-cultural gerontology, 35(3), 237-254. https://doi.org/10.1007/s10823-020-09406-8

Capella, C. (2011). Hacia narrativas de superación: El desafío para la psicoterapia con adolescentes de integrar la experiencia de agresión sexual a la identidad personal [Tesis Doctoral, Universidad de Chile]. Repositorio Académico de la Universidad de Chile. https://repositorio.uchile.cl/handle/2250/112610

Capella, C. (2013). Una propuesta para el estudio de la identidad con aportes del análisis narrativo. Psicoperspectivas, 12(2), 117-128.

Cardona, A. M. A., \& Salgado, S. V. A. (2015). Investigación narrativa: apuesta metodológica para la construcción social de conocimientos científicos. CES Psicología, 8(2), 171-181.

Cassady, K., Ruitenberg, M. F. L., Reuter-Lorenz, P. A., Tommerdahl, M., \& Seidler, R. D. (2020). Neural dedifferentiation across the lifespan in the motor and somatosensory systems. Cerebral Cortex, 30(6), 3704-3716. https://doi. org/10.1093/cercor/bhz336

Comisión Económica para América Latina y el Caribe. (2015). Anuario Estadístico de América Latina y el Caribe 2015. Naciones Unidas.

D'cruz, M., \& Banerjee, D. (2020). "An invisible human rights crisis": The marginalization of older adults during the COVID-19 pandemic - An advocacy review. Psychiatry research, 292, 113369. https://doi.org/10.1016/j.psychres.2020.113369

da Silva, R., Afonso, P., Fonseca, M., \& Teodoro, T. (2020). Comparing sleep quality in institutionalized and non-institutionalized elderly individuals. Aging \& Mental Health, 24(9), 1452-1458. https://doi.org/10.1080/13607863.2019.1619168 
Elsherbiny, M. M. K., \& Al Maamari, R. H. (2018). The Effectiveness of Logotherapy in Mitigating the Social Isolation of Neglected Institutionalised Older People. British Journal of Social Work, 48(4), 1090-1108. https://doi.org/10.1093/bjsw/bcy043

Flecha, A. C. (2019). Autoaceptación y sentido/propósito vital en personas mayores institucionalizadas. Pedagogía Social. Revista Interuniversitaria, 33, 139-151. https://doi.org/10.7179/PSRI_2019.33.10

Flick, U. (2004). Introducción a la investigación cualitativa. Morata.

Franco, M. (2018). Desempeño ocupacional, bienestar psicológico y sentido de la vida en personas institucionalizadas. Estudio preliminar. Revista de Psicología de la Salud, 6(1), 87-123.

Frankl, V. (1979). El hombre en busca del sentido. Herder.

Frankl, V. (1982). Psicoanálisis y Existencialismo. De la Psicoterapia a la Logoterapia. Fondo de Cultura Económica.

Frankl, V. (1983). El hombre doliente. Fundamentos Antropológicos de la Psicoterapia. Herder.

Frankl, V. (1995). La psicoterapia al alcance de todos. Herder.

Freitas, M., \& Scheicher, M. (2010). Qualidade de vida de idosos institucionalizados. Revista Brasileira de Geriatria e Gerontologia, 13(3), 395-401. https://doi.org/10.1590/ S1809-98232010000300006

Guillen, F., \& Elida, D. (2019). Investigación cualitativa: Método fenomenológico hermenéutico. Propósitos y Representaciones, 7(1), 201-229. https://doi. org/10.20511/pyr2019.v7n1.267

Hill, P., \& Turiano N. (2014). Purpose in Life as a Predictor of Mortality Across Adulthood. Psychological Science, 25, 1482-1486. https://doi.org/10.1177/0956797614531799

Howell, B. M., \& Peterson, J. R. (2020). "With Age Comes Wisdom:" a Qualitative Review of Elder Perspectives on Healthy Aging in the Circumpolar North. Journal of crosscultural gerontology, 35(2), 113-131. https://doi.org/10.1007/s10823-020-09399-4

$\mathrm{Ju}, \mathrm{H}$. (2017). The relationship between physical activity, meaning in life, and subjective vitality in community-dwelling older adults. Archives of Gerontology \& Geriatrics, 73, 120-124. https://doi.org/10.1016/j.archger.2017.08.001

Ju, H., Shin, J., Kim, C., Hyun, M., \& Park, J. (2013). Mediational effect of meaning in life on the relationship between optimism and well-being in community elderly. Archives of Gerontology and Geriatrics, 56(2), 309-313. https://doi.org/10.1016/j. archger.2012.08.008

Kim, E., Sun, J., Park, N., Kubzansky, L., \& Peterson, C. (2013). Purpose in life and reduced risk of myocardial infarction among older U.S. adults with coronary heart disease: A two-year follow-up. Journal of Behavioral Medicine, 36, 124-133. https://doi. org/10.1007/s10865-012-9406-4

Krause, N. (2009). Meaning in life and mortality. Journal of Gerontology: Social Sciences, 64B(4), 517-527. https://doi.org/10.1093/geronb/gbp047

Lang, F. R., Albert, I., \& Kliegel, M. (2020). Psychology and aging: European perspectives. European Psychologist, 25(3), 159-161. https://doi.org/10.1027/1016-9040/a000411 
Längle, A. (2008). Vivir con Sentido. Aplicación práctica de la Logoterapia. Lumen.

Moreira, P., Carneiro, A., Lima, A., \& Barbosa, L. (2016). Quality of Life of Institutionalized Elderly in Brazil. Social Indicators Research, 126(1), 187-197. https://doi.org/10.1007/s11205-015-0888-7

Organización Mundial de la Salud. (2017). La salud mentaly los adultos mayores. https://www. who.int/es/news-room/fact-sheets/detail/la-salud-mental-y-los-adultos-mayores

Park, C., \& Folkman, S. (1997). Meaning in the context of stress and coping. Review of General Psychology, 30, 115-144. https://doi.org/10.1037/1089-2680.1.2.115

Reich, A. J., Claunch, K. D., Verdeja, M. A., Dungan, M. T., Anderson, S., Clayton, C. K., Goates, M. C., \& Thacker, E. L. (2020). What Does "Successful Aging" Mean to you? - Systematic Review and Cross-Cultural Comparison of Lay Perspectives of Older Adults in 13 Countries, 2010-2020. Journal of cross-cultural gerontology, 35, 445-478. https://doi.org/10.1007/s10823-020-09416-6

Riessman, C. (2008). Narrative Methods for the Human Sciences. Sage.

Servicio Nacional del Adulto Mayor. (2013). Política integral de envejecimiento positivo para Chile 2012-2025. SENAMA. https://www.mop.cl/GIS/Documents/SENAMA.pdf

Sexton, C. E., Betts, J. F., Dennis, A., Doherty, A., Leeson, P., Holloway, C., Dall'Armellina, E., Winkler, A. M., Demnitz, N., Wassenaar, T., Dawes, H., \& Johansen-Berg, H. (2020). The effects of an aerobic training intervention on cognition, grey matter volumes and white matter microstructure. Physiology \& Behavior, 223, 2-8. https://doi. org/10.1016/j.physbeh.2020.112923

Silva, J., Albuquerque, M. C., Souza, E. M., Monteiro, F. S., \& Esteves, G. G. (2015). Sintomas Depressivos e Capacidade Funcional em Idosos Institucionalizados. Cultura de los cuidados, 19(41), 157-167. https://doi.org/10.14198/cuid.2015.41.18

Steger, M., Shigehiro, O., \& Kashdan, T. (2009). Meaning in life across the life span: Levels and correlates of meaning in life from emerging adulthood to older adulthood. The Journal of Positive Psychology, 4(1), 43-52. https://doi. org/10.1080/17439760802303127

Strauss, A., \& Corbin, J. (2002). Bases de la investigación cualitativa. Técnicas y procedimientos para desarrollar la Teoría Fundamentada. Universidad de Antioquia.

Theurer, K., Mortenson, W., Stone, R., Suto, M., Timonen, V., \& Rozanova, J. (2015). The need for a social revolution in residential care. Journal Aging Stud, 35, 201-210. https://doi.org/10.1016/j.jaging.2015.08.011

Timbers, V. L., \& Childers, M. (2020). A case study in group spiritual care for residents of a post-acute care facility. Journal of Religion, Spirituality \& Aging. https://doi.org/1 0.1080/15528030.2020.1822262

Tiwari, S., Singh, R., \& Chand, H. (2016). Spirituality and Psychological Wellbeing of Elderly of Uttarakhand: A Comparative Study Across Residential Status. Journal of Psychology, 7(2), 112-118. https://doi.org/10.1080/09764224.2016.11907851

Usta, E., \& Bayram, S. (2021). Determination of Factors Affecting Self-Transcendence and Meaning in Life Among the Elderly Receiving Institutional Care. Journal of Religion \& Health, 60(3), 2024-2036. https://doi.org/10.1007/s10943-020-01165-7

Vial, W. (2000). La antropología de Viktor Frankl:el dolor, una puerta abierta. Editorial Universitaria. 
Volkert, J., Härter, M., Dehoust, M., Ausín, B., Canuto, A., da Ronch, C., Suling, A., Grassi, L., Munoz, M., Santos-Olmo, A., Sehner, S., Weber, K., Wegscheider, K., Wittchen, H., Schulz, H., \& Andreas, S. (2017). The role of meaning in life in communitydwelling older adults with depression and relationship to other risk factors. Aging \& Mental Health, 23(1), 100-106 https://doi.org/10.1080/13607863.2017.1396576

Wiles, J. (2005). Conceptualizing place in the care of older people: the contributions of geographical gerontology. Journal of Clinical Nursing, 14(s2), 100-108. https://doi. org/10.1111/j.1365-2702.2005.01281.x

Worrall, C., Jongenelis, M., \& Pettigrew, S. (2020). Modifiable protective and risk factors for depressive symptoms among older community-dwelling adults: A systematic review. Journal of Affective Disorders, 272, 305-317. https://doi.org/10.1016/j. jad.2020.03.119 